\title{
Evaluation of a tailored implementation strategy to improve the management of patients with chronic obstructive pulmonary disease in primary care: a study protocol of a cluster randomized trial
}

\author{
Maciek Godycki-Cwirko ${ }^{1 *}$, Izabela Zakowska', Katarzyna Kosiek', Michel Wensing², Jaroslaw Krawczyk \\ and Anna Kowalczyk ${ }^{1}$
}

\begin{abstract}
Background: Chronic obstructive pulmonary disease (COPD) remains a major health problem, strongly related to smoking. Despite the publication of practice guidelines on prevention and treatment, not all patients with the disease receive the recommended healthcare, particularly with regard to smoking cessation advice where applicable. We have developed a tailored implementation strategy for enhancing general practitioners' adherence to the disease management guidelines. The primary aim of the study is to evaluate the effects of this tailored implementation intervention on general practitioners' adherence to guidelines.
\end{abstract}

Methods/Design: A pragmatic two-arm cluster randomized trial has been planned to compare care following the implementation of tailored interventions of four recommendations in COPD patients against usual care. The study will involve 18 general practices ( 9 in the intervention group and 9 in the control group) in Poland, each with at least 80 identified (at the baseline) patients with diagnosed COPD. The nine control practices will provide usual care without any interventions. Tailored interventions to implement four recommendations will be delivered in the remaining nine practices. At follow-up after nine months, data will be collected for all 18 general practices. The primary outcome measure is physicians' adherence to all four recommendations: brief anti-smoking advice, dyspnea assessment, care checklist utilization and demonstration to patients of correct inhaler use. This measurement will be based on data extracted from identified patients' records. Additionally, we will survey and interview patients with chronic obstructive pulmonary disease about the process of care.

Discussion: The results of this trial will be directly applicable to primary care in Poland and add to the growing body of evidence on interventions to improve chronic illness care.

Trial registration: This trial has been registered with Clinical Trials Protocol Registration System. Trial number: NCT01893476.

Keywords: Chronic obstructive pulmonary disease, Implementation science, Primary health care

\footnotetext{
* Correspondence: maciej.godycki-cwirko@umed.lodz.pl

${ }^{1}$ Centre for Family and Community Medicine, Medical University of Lodz, 20 Kopcinskiego Street, Lodz 90-153, Poland

Full list of author information is available at the end of the article
} 


\section{Background}

Chronic obstructive pulmonary disease (COPD) remains a major health problem. Worldwide it has been ranked as the sixth leading cause of death for both genders [1]. In 2020 COPD is projected to rank fifth worldwide in burden of disease. It is also projected to be the fourth leading cause of death worldwide by 2030 due to an increase in smoking rates and demographic changes in many countries [2]. A national survey conducted in Poland in 2007 indicated that $34 \%$ of men smoked daily, $2 \%$ were occasional smokers, $19 \%$ were former smokers and $45 \%$ had never smoked. In women these percentages were $23,3,10$ and $64 \%$ respectively [3].

Epidemiological studies of COPD on a representative sample have not been performed in Poland, but estimates from smaller studies suggested a relatively high prevalence. Studies in selected geographical areas found that signs and symptoms of COPD were seen in about $10 \%$ of patients over 40-years old [4]. Studies in large cities in Poland showed a $9.8 \%$ prevalence of COPD in populations between 41 and 72 years old [5]. These data are similar to other European data, describing prevalence rates of 4 to $11 \%$ in adults in Europe [6]. The total number of people suffering from COPD in Poland is estimated to be about 2 million (on a population of 38 million inhabitants). This places COPD as the third most frequent chronic illness, and it is the fourth most common cause of death in Poland [7].

In Poland, most COPD patients are treated in primary care, which is the entry point to the public health care system. The system is based on compulsory health insurance, managed by the National Health Fund (NHF), which purchases health services from physicians and health care enterprises. Patients register with a particular primary care practice, signing on to an individual general practitioner's list of patients, and can be referred for specialist outpatient consultation or to a hospital, if needed. Ambulatory care (primary and out-patient specialist services) is provided by therapeutic entities (clinics or dispensaries) and by medical practices. Rehabilitation and long-term care are provided within both the health care sector and the social care sector, but coordination between the two is poor. Furthermore, limited financial resources available to the NHF and shortages of medical personnel have negative effects on access to health care services [8].

There is no national consensus on the care paths for COPD, and various guidelines on COPD are used, some of which national and some international $[9,10]$. However, the guidelines in use share most of recommendations that we have identified and prioritized for the purpose of this trial. There is very little evidence on the degree of adherence to the guidelines for the management of COPD used by Polish physicians. One study, which evaluated the NHF funded COPD prevention program aiming to reduce the incidence and disability related to COPD by complex educational, diagnostic and therapeutic interventions in people at risk, showed low rates of participation of primary health care providers despite additional funding. An interesting finding was that the extent of program completion was strongest for qualified general practitioners compared to physicians without specialty training or non-generalists: internal diseases specialists and pediatricians working at primary care setting [11].

It is unclear whether studies on the implementation of evidence-based recommendations for primary care in COPD patients from countries with well-developed primary care systems, like the United Kingdom and the Netherlands $[12,13]$ can be translated to Poland. In searching the Polish medical literature, we did not find published research on the implementation of COPD guidelines or recommendations in Poland. The authors identified three papers with results partially related to the subject of this study [14-16]. These found that $30 \%$ of patients with the diagnosis do not fulfill criteria for COPD, in 15\% of cases spirometry had not been performed, and more than $70 \%$ of patients received inhaled steroids. Such findings suggest that adherence to diagnostic and management recommendations are inadequate.

Preliminary qualitative evidence from the authors' earlier work showed that not one single guideline for COPD was used by all GPs or medical specialists. However, responders pointed out the individual recommendations most commonly used and the barriers to their implementation. The identified barriers for adhering to specific recommendations were: (1) lack of knowledge on smoking cessation brief intervention, (2) dyspnea evaluation tool was unavailable, (3) lack of a care plan, and (4) lack of demonstration-inhaling devices. Other research has indicated that the treatment parameters from the (COPD) guidelines are not always measured [17-19]. The consequence is that not all patients receive recommended advice and treatment from their physicians.

This study is part of the Tailored Implementation for Chronic Diseases (TICD) international collaborative research project, which is being undertaken in Germany, Norway, Poland, the Netherlands and the United Kingdom. The aim is to develop and test methods of tailoring knowledge-implementation interventions to determinants of practice in chronic illness care [20]. In Poland, the focus is on the implementation of COPD guidelines in primary care. The research group in Poland selected four key recommendations applicable to primary care (Table 1) from the four COPD guidelines most relevant to primary care and used in Poland.

\section{Objective}

The objective of this study is to examine the effectiveness of a tailored implementation strategy for enhancing 


\section{Table 1 Key recommendations for COPD management}

A Brief smoking cessation counseling is effective and every tobacco user should be offered stop-smoking advice at every contact with health care providers.

B The prognosis of COPD should be estimated using the mMRC dyspnea scale.

C The patient should be given the basic information about his/her disease, its treatment and the expected effects of applied drugs, in order to enhance that the patient takes an active, participating role in the long-term treatment.

D The patient should be trained in the correct use of all devices which he/she will use for inhalation.

physicians' adherence to four recommendations for the management of COPD patients in primary care. The process of tailoring implementation interventions and the impact on the effectiveness of the strategy will also be studied.

\section{Research questions}

The questions we will be attempting to address in this study are as follows: what is the effectiveness of a tailored implementation strategy compared to usual care in enhancing primary care physicians' adherence to guidelines?, and how do the determinants of practice targeted by the tailored implementation strategy change over time, and how does their variability relate to the effectiveness of implementation strategies?

\section{Methods/design \\ Study design}

This study is a two-arm pragmatic, cluster randomized trial (CRCT) [21,22], which compares a tailored intervention program directed at general practitioners with usual primary care. It is localized in Lodz, the third largest city in Poland, with a population of about 750.000 inhabitants with broadly average mortality and morbidity, although specific health data are lacking. It aims to include general practices with adult patients with COPD under their care. The general practices will be randomized into two equally sized groups

\section{Sample size calculation}

The sample size calculation indicated that, for a two-arm cluster controlled randomized trial, a minimum number of 16 primary care practices with minimum of $30 \mathrm{COPD}$ patients per practice is required. A total of 16 clusters and at least 480 subjects is required at baseline and follow-up (8 clusters in each study arm) to detect a difference, or change in adherence, of between $40 \%$ and $60 \%$ with selected COPD recommendations, between the intervention group (facilitating adherence to guidelines) and the control group without intervention (usual care), with $80 \%$ power, a two-tailed alpha of 0.05 , and intra-cluster correlation of 0.05 .
A standard sample size formula was used to calculate the initial unadjusted sample size requirements, followed by appropriate adjustment for clustering by general practice according to Campbell et al. [23], with expected small clustering effect $(\mathrm{ICC}=0.05)$. Previous reported ICCs vary from 0.03 for smoking advice [23] to 0.05 for cluster randomized trials in primary care (data from Trial of Older People in the Community) [24,25]. It was decided to increase the sample by $10 \%$ to account for contingencies such as non-response or recording error, giving a total of 18 practices.

With regard to the number of patients with COPD per practice, an assumed response rate of 58\% [26,27] and a dropout of $35 \%$ [28], which led to a total of 80 COPD patients needed per general practice at baseline. Patients will be selected via the medical records using ICD code J44, and medical records will be labelled at the baseline of the study.

\section{Setting and participants General practices}

Eighteen general practices selected with random sampling within the Lodz region of Poland, with 80 or more registered COPD patients each (identified via J44 ICD-10), will be invited to participate in the study. The baseline number of COPD patients is determined by assumed response rate and dropout described above, to assure at least 30 at follow-up. Some of the practices were previously involved in other studies and have an established agreement with the Medical University of Lodz in the field of research and vocational training. The main study has been planned to take place between December 2013 and September 2014. After having given informed consent, primary care practices will be randomly allocated to one of two groups. The medical records of patients with COPD will be labelled with blue stickers at the baseline of the study (Figure 1).

\section{General practitioners}

All GPs working in targeted practices are required to participate in the study.

\section{Eligibility criteria \\ Patients}

Eligible patients will be approached at follow-up. Only patients who were treated in the same practice at baseline (with the labelled medical records) will be considered for inclusion so that the study is prospective. Exclusion criteria are: (1) terminal illness and (2) cognitive impairments. At follow up 80 patients, randomly selected (with personal data unknown to researchers) will receive an informed consent form and an invitational letter via their GPs, and will be asked to fill in a questionnaire and/or participate in an audio taped interview or focus group. This letter will provide comprehensive information about 


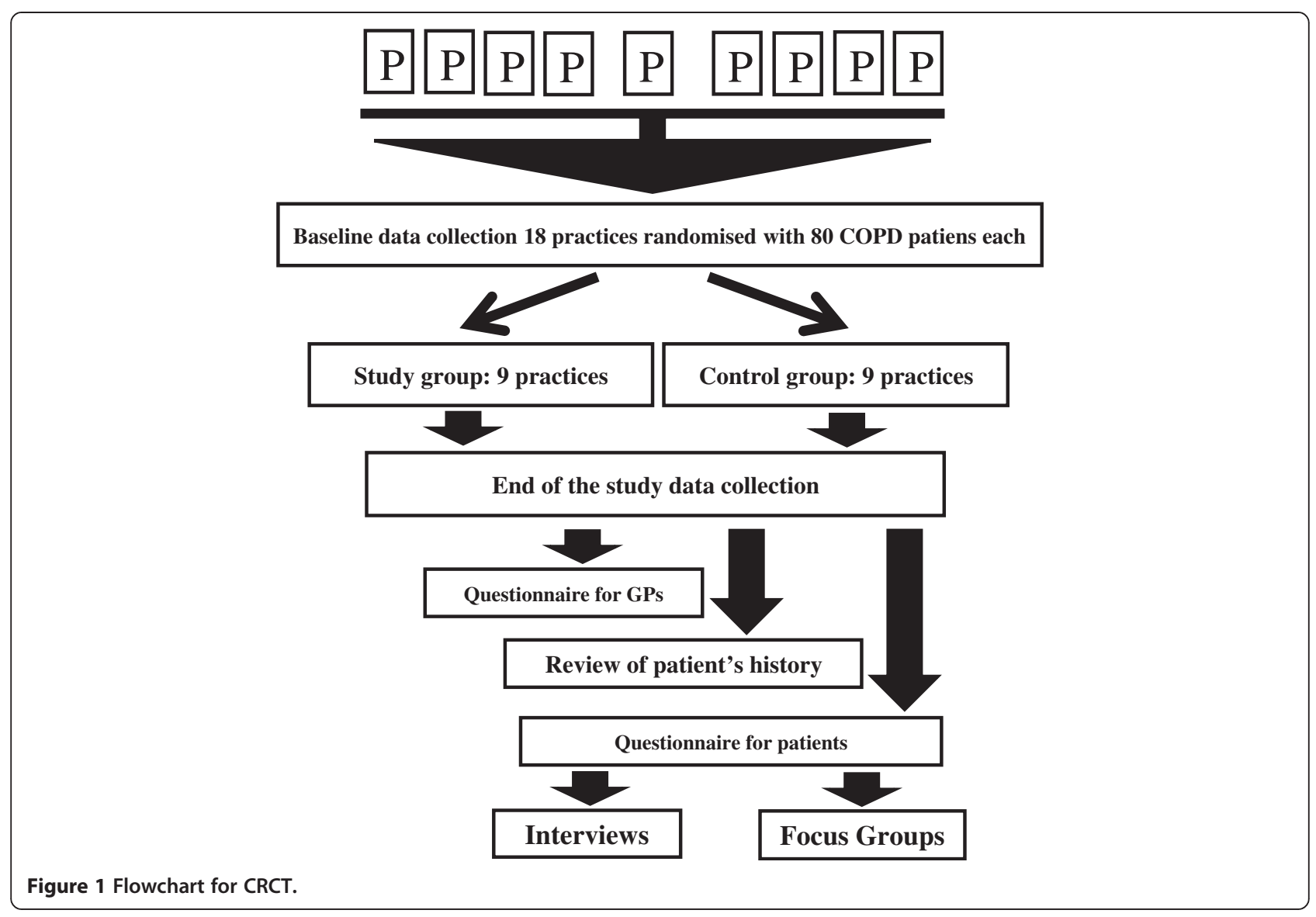

the study. Contact details of the researchers will be provided so that patients can ask questions. Patients' information gathered from questionnaires or from interviews will be anonymized.

\section{Randomization}

Randomization will be performed by a statistician not involved in the trial, through a computer. The general practices will be allocated randomly to two equally sized groups: an intervention group and a control group. At the end of the study a group of patients will be randomly selected in order to invite them to fill out the end-ofstudy questionnaire. Participants in this trial will not be blinded. Descriptive statistics and logistic regression will be used for analyzing the data. The level of statistical significance will be $p<0.05$.

\section{Blinding}

COPD patients within each participating intervention and control practice will be identified at the baseline. Because of the nature of the intervention, it is not possible to blind GP participants (in practices).

Outcome assessment will not be blinded as the research assistants will be aware of practice group allocation, and the data analysis will be performed by researchers and a statistician blinded to the study group.

\section{Implementation program}

The implementation program is based on multiphase research performed in the TICD project. In the first phase has identified barriers and enablers for improving COPD care, focusing on the four recommendations selected. One hundred and sixty determinants were listed, grouped (according to the TICD checklist domains [29]), and judged by our research team on importance and changeability using Likert scales. The result was 24 determinants of practice. The second phase of the project, identified determinants were prioritized during focus group discussions and matched to implementation strategies. The final decisions on the interventions matched to the determinants for four recommendations are presented in Table 2. This procedure led to the following implementation program.

\section{Smoker identification and brief intervention}

First, participating physicians will receive training in brief smoking status identification and anti-smoking counselling, and will be asked to record information about the actions they perform in patients' medical records. This intervention will address the recommendation of Global 
Table 2 Identified determinants and interventions for selected recommendations

\begin{tabular}{|c|c|c|c|}
\hline & Recommendation & Determinant & Proposed intervention(s) \\
\hline $\bar{A}$ & A brief clinician's counseling to quit smoking & Additional training for GPs & $\begin{array}{l}\text { Labeling medical records for COPD patients and training GPs } \\
\text { in brief intervention }\end{array}$ \\
\hline B & $\begin{array}{l}\text { The prognosis of COPD should be estimated } \\
\text { using mMRC dyspnea scale }\end{array}$ & Prepared form & $\begin{array}{l}\text { Additional form (in paper or in computer medical records) with } \\
\text { mMRC scale }\end{array}$ \\
\hline$C$ & $\begin{array}{l}\text { The patient should be given the basic } \\
\text { information about his/her disease }\end{array}$ & Checklist in medical history & $\begin{array}{l}\text { Providing physicians with a checklist in patients' records to facilitate } \\
\text { what should be done during consultation }\end{array}$ \\
\hline $\mathrm{D}$ & $\begin{array}{l}\text { The patient has to be trained in the correct } \\
\text { use of all devices }\end{array}$ & Inhaling devices for training & $\begin{array}{l}\text { To provide GPs' clinics with training inhaler devices to show } \\
\text { patient's how to use them }\end{array}$ \\
\hline
\end{tabular}

Initiative for Chronic Obstructive Lung Disease (GOLD) based on Wilson et al. [30].

\section{Dyspnea evaluation}

Second, an additional form containing the modified Medical Research Council Dyspnea Scale (mMRC) will be inserted into patients' medical records on paper. It is a validated patient symptom questionnaire for a subjective assessment of COPD symptoms. This intervention will address the recommendation of National Institute for Health and Care Excellence based on Fletcher et al. [31].

GPs will be asked to determine the patient's status according to the scale and put this information into the patient's medical records.

\section{COPD check list}

Third, a checklist for practitioners will be provided with information about what should be done while consulting on a patient with COPD. This intervention will address the recommendation of the European Respiratory Society [32]. It will cover points such as that a patient should be given basic information about COPD, its treatment, and the expected effects of applied drugs, making the patient an active, aware participant in their long-term treatment. GPs will be asked to provide patients with the information and tick a box if it is done.

\section{Demonstration inhaler devices}

Finally, practices will be provided with training inhaler device sets for health care staff and train GPs on how to instruct patients to use devices properly. This intervention will address the recommendation of the Polish Society of Lung Diseases [33].

GPs will be asked to teach patients in the correct use of each device and record information about the fact in patient's medical records.

\section{Control group}

In this arm, GPs will provide their usual care for COPD patients. The practices in the control group will receive feedback after the end of the study about their own performance in comparison with the performance of other practices in the study in relation to the guideline recommendations. The GPs will receive no intervention during the study.

\section{Outcomes/measures}

\section{Primary outcome}

The primary outcome will be the GPs' adherence to the recommendations, which is dichotomized as follows. A positive score is given if all recommendations are followed, while following less than four recommendations is given a negative score. Data listed in Table 3 will be obtained from the patients' medical records (independent of the checklist that is provided as part of the intervention) and during interviews. All identified COPD patients who gave informed consent in each practice will be included, and full data protection procedures will be followed.

\section{Secondary outcomes}

The secondary outcome will be patient reported health status. We will review COPD patients' medical records to measure health outcomes such as a change in smoking status, the quantity of COPD medications prescribed, dyspnea perception and number of exacerbations in the past and over the study period.

\section{Process evaluation}

All aspects of the coordinated process evaluation will be implemented in this study, following the international study protocol for the TICD project, in order to identify determinants of change in chronic illness care, to examine the validity of the tailoring methods that were applied, and to analyze the association of implementation activities with the effectiveness of the program [34]. The process evaluation will comprise three main components: a structured survey with health professionals in the trials, semi-structured interviews with a purposeful sample of this study population, and standardized documentation of organizational practice characteristics. The evaluation will be guided by 'logic models' of the implementation programs: frameworks that specify the linkages between the strategies used, the determinants addressed by tailoring, and the anticipated outcomes.

A written survey will be undertaken involving participating health professionals. The questionnaire will list the 


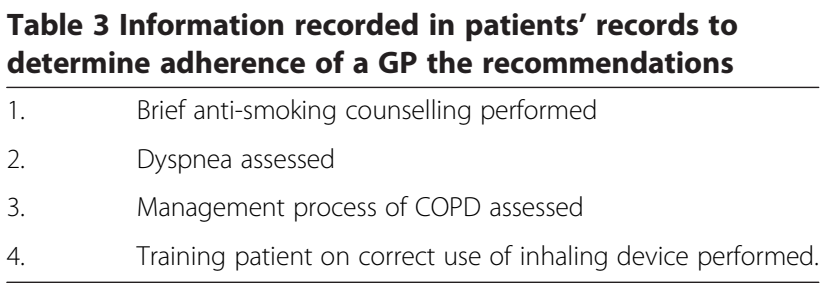

determinants of practice, which were identified and prioritized in an earlier phase of the TICD project, respondents being asked to assess whether the program successfully targeted them. A free text field will be used to identify possible other determinants. The survey will also contain questions on the actual implementation activities that took place, to record the extent to which the target group used the offered interventions, and any adaptions made in the delivery phase of the implementation program. The core components of the implementation program will be specified. For each of the core components, content, duration, frequency and coverage will be recorded in a structured way. The content, duration, frequency and coverage aspects of intervention fidelity will also be covered. A free text field will be added to identify strategies which have been missed in the tailoring process, allowing the evaluation of the methods used for tailoring.

The face-to-face or telephone interviews will be performed with a purposive sample of health professionals. Interim analysis after five to ten interviews will be performed to adapt the interview format and purposeful sampling scheme as required.

\section{Data collection}

At the end of the study data will be collected by review of medical records of COPD patients identified at the baseline who visited their GPs during the last nine months. Extracted information will cover the following: brief anti-smoking advice performed, information about dyspnea, COPD checklist utilization and training on using inhalers.

Patients with COPD who have given signed informed consent will be asked to fill in a questionnaire and participate in an interview containing questions about the process of care, information received from GPs and patients' health status perception.

\section{Statistical methods}

The primary analysis will be on an intention to treat basis. Missing values will be inputted with a multiple imputation method. Data will be coded, cleaned and locked before any analyses are made. Quantitative data will be analyzed in aggregate, using the Statistical Package for the Social Sciences (SPSS, (version 17, IBM Corp.) and Statistica (v10, StatSoft Inc.). Study groups will be compared with respect to the determinants of COPD and its improvement. Only members of the research team who need access to the database to fulfill their roles within the study will be granted access to the database. Demographic characteristics of the practices and the participants within the practices will be described using percentages, measures of central tendency (means or medians) and measures of variation (standard deviations or ranges). The analysis will be based on the patients nested within practices two-level model. The primary endpoint is the GPs adherence to four COPD guidelines after nine months, in each practice. Secondary endpoints are the change in smoking status and health care utilization during the nine months amongst included patients. Data will be compared between arms using logistic regression (with logit link function and binomial distribution) with patient at level one and practice at level two. The ICC and odds ratios and $95 \%$ confidence intervals will be estimated using twolevel random intercepts logistic regression models. The significance level will be set at $\alpha=0.05$ for all analyses.

\section{Ethical approval}

This study has been approved by the Bioethical Committee of Medical University of Lodz, Poland (reference RNN/ 491/13/KB of 18 June 2013).

\section{Discussion}

COPD care improvement in primary care has not been studied extensively. Schermer et al. observed a small and late effect of e-learning and repeated feedback on the quality of spirometry as performed by family practice nurses [35]. Kennedy et al. studied at practice-level training in a whole systems approach to self-management support for patients with chronic conditions and found no statistically significant differences between patients attending trained practices and those attending control practices, although this study looked at three conditions simultaneously (diabetes, COPD and irritable bowel syndrome) [36]. While these trials focused on specific aspects of COPD care (spirometry and self-management education, respectively), the intervention program in this study intends to improve COPD care more broadly.

Meulepas et al. studied the effect of a primary care model that included smoking cessation, inhaler technique training and dyspnea scoring according to the MRC scale [12]. V an den Bemt et al. also studied guideline based COPD-management in primary care [13]. However their interventions were at least partly directed at patients, while this study targets general practitioners. This study is probably the first randomized trial of a tailored implementation program for improving primary care for patients with chronic illness in an East European country. The study has some limitations, such as possible selection bias since practices with less than 80 COPD patients were excluded as well as those that have some GPs teachers. 
Nevertheless, it is the authors' belief that the results will be directly relevant and applicable to primary care in Poland. If the implementation program is effective, then wide-scale application would be warranted.

\section{Trial status}

Registration has been completed, general practices have been recruited and randomized, and interventions delivered.

\section{Abbreviations}

ATS: American Thoracic Society; COPD: chronic obstructive pulmonary disease; CRCT: Cluster Randomized Controlled Trial; ERS: European Respiratory Society; EU: European Union; GOLD: Global Initiative for Chronic Obstructive Lung Disease; GP: General Practitioner; ICC: Intracluster Correlation Coefficient; ICD: International Classification of diseases; mMRC: Modified Medical Research Council; NHF: National Health Fund; NICE: National Institute on Health and Clinical Excellence; P: practice; PSLD: Polish Society on Lung Diseases; SPSS: Statistical Package for the Social Sciences; TICD: Tailored Implementation for Chronic Diseases.

\section{Competing interests}

The authors declare that they have no competing interests.

\section{Authors' contributions}

MG-C and JK developed the idea and wrote the draft version of this protocol, which was commented by AK, IZ and KK. IZ did the sample size calculation. M-C corrected and rewrote consecutive versions. All authors critically assessed and approved this study protocol. Project partners discussed with and reviewed the protocol. MW revised and commented on draft versions and reviewed the final version. All authors have read and approved the manuscript for publication.

\section{Authors' information}

TICD is a collaborative project between five European countries (The Netherlands, UK, Germany, Poland and Norway). Michel Wensing from Radboud University, the Netherlands is the principal investigator in the TICD project. This TICD WP4 COPD trial in Poland will be coordinated by Maciek Godycki-Cwirko at the Medical University of Lodz. The researchers in the Polish team have experience with cluster randomized trials.

\section{Acknowledgements}

The authors thank TICD project partners who critically reviewed and commented the protocol. Special thanks are addressed to Professor Richard Baker, who proofread the final version and provided linguistic corrections. The study is funded by the European Union 7 Framework Program within the theme HEALTH.2010.3.1-1 under grant agreement no 258837.

\section{Author details}

${ }^{1}$ Centre for Family and Community Medicine, Medical University of Lodz, 20 Kopcinskiego Street, Lodz 90-153, Poland. ${ }^{2}$ Radboud University Medical Centre, Scientific Institute for Quality of Healthcare, P.O. Box 9101, 114, Nijmegen 6500, HB, The Netherlands.

Received: 31 December 2013 Accepted: 19 March 2014 Published: 4 April 2014

\section{References}

1. Mathers $C D$, Loncar D: Projections of global mortality and burden of disease from 2002 to 2030. Plos Med 2006, 3:e442.

2. World Health Statistics: Chapter 1 - burden: mortality, morbidity and risk factors; 2008. http://www.who.int/nmh/publications/ncd_report_chapter1. pdf. Global status report on noncommunicable diseases 2010, World Health Organization Press, printed in Italy.

3. Bednarski B, Gorynski P, Lata E, Parchimowicz T, Przewozniak K, Wojtyniak B: The current status of the tobacco epidemic in Poland. World Health Organization regional office for Europe. Copenhagen: WHO Regional Office for Europe; 2009. http://www.euro.who.int/_data/assets/pdf_file/0006/ 68064/E92470.pdf.
4. Niepsuj GK, Niepsuj K, Ziora D, Polonska A, Cieslicki J: Przewlekla obturacyjna choroba pluc wsrod mieszkancow miasta Zabrze. [Chronic obstructive pulmonary disease among residents of the city of Zabrze]. Wiad Lek 2002, 55:354-359.

5. Plywaczewski R, Bednarek M, Jonczak L, Zielinski J: Czestosc wystepowania POCHP wsrod mieszkancow prawobrzeznej Warszawy. [Prevalence of COPD in Warsaw population]. Pneumonol Alergol Pol 2003, 71:329-335.

6. European Respiratory Society/European Lung Foundation: Chronic obstructive pulmonary disease. In European lung white book - the first comprehensive survey on respiratory health in Europe. Edited by Loddenkemper R, Gibson GJ, Sibille Y. Sheffield: ERSJ; 2003:34-43.

7. Czermak G, Głaz M, Głowacka-Smolis K, Lipowska E, Marikin M, Ostrowska A, Pilaszek K, Rybak-Nguyen E, Szczepańska A, Szydłowska G: Rocznik statystyczny Rzeczpospolitej Polskiej. [Statistical yearbook of the Republic of Poland]; 2008:204. Statistical Publishing Establishment, Warsaw, Poland. Warsaw. 2008.

8. Sagan A, Panteli D, Borkowski W, Dmowski M, Domanski F, Czyzewski M, Gorynski P, Karpacka D, Kiersztyn E, Kowalska I, Ksiezak M, Kuszewski K, Lesniewska A, Lipska I, Maciag R, Madowicz J, Madra A, Marek M, Mokrzycka A, Poznanski D, Sobczak A, Sowada C, Swiderek M, Terka A, Trzeciak P, Wiktorzak K, Wlodarczyk C, Wojtyniak B, Wrzesniewska-Wal I, Zelwianska D, et al: Poland health system review. Health Syst Transit 2011, 13:1-193.

9. van den Bemt $L$, Schermer T, van Weel $C$ : Rational monitoring of COPD: where do current clinical guidelines stand? Eur Respir J 2007, 29:1078-1081. doi:10.1183/09031936.00043107.

10. Miravitlles M, Soler-Cataluña JJ, Calle M, Molina J, Almagro P, Quintano JA, Riesco JA, Trigueros JA, Piñera P, Simón A, López-Campos JL, Soriano JB, Ancochea J, Spanish Society of Pulmonology and Thoracic Surgery: Spanish COPD Guidelines (GesEPOC): pharmacological treatment of stable COPD. Spanish Society of Pulmonology and Thoracic Surgery. Arch Bronconeumol 2012, 48:247-257.

11. Godycki-Cwirko M, Koziarska-Rosciszewska M, Kosiek K: The delivery of prevention programmes for cardiovascular disease and chronic obstructive pulmonary disease in Lodz by primary care physicians. Archives of Medical Science 2010, 6:208-213.

12. Meulepas MA, Jacobs JE, Smeenk FW, Smeele I, Lucas AE, Bottema BJ, Grol RP: Effect of an integrated primary care model on the management of middle-aged and old patients with obstructive lung diseases. Scand J Prim Health Care 2007, 25:186-192.

13. van den Bemt L, Schermer TR, Smeele IJ, Boonman-de Winter LJ, van Boxem T, Denis J, Grootens-Stekelenburg JG, Grol RP, van Weel C: An expert-supported monitoring system for patients with chronic obstructive pulmonary disease in general practice: results of a cluster randomized controlled trial. Med J Aust 2009, 191:249-254.

14. Mejza F, Nizankowska-Mogilnicka E, Kurzawa R, Gorski P, Wirkijowski B, Jaeschke R: Outpatients specialist care of chronic obstructive pulmonary disease patients in Poland - results of the KOMPAS study. Pneumonol Alergol Pol 2009, 77:507-516.

15. Zielinski J, Roszkowski-Sliz K: Narodowy program wczesnego rozpoznawania i profilaktyki POChP [National program of early diagnoais and prevention of COPD]. Pneumonol Alergol Pol 2002, 70:125-129.

16. Zielinski J, Kuca P, Ptasznik P, Plywaczewski R: Management of COPD: pulmonologists' adherence to Polish guidelines. Pneumonol Alergol Pol 2005, 73:135-141.

17. Jones RC, Dickson-Spillmann M, Mather MJ, Marks D, Shackell BS: Accuracy of diagnostic registers and management of chronic obstructive pulmonary disease: the Devon primary care audit. Respir Res 2008, 9:62.

18. Rutschmann OT, Janssens JP, Vermeulen B, Sarasin FP: Knowledge of guidelines for the management of COPD: a survey of primary care physicians. Respir Med 2004, 98:932-937.

19. Schneider A, Gantner L, Maag I, Borst MM, Wensing M, Szecsenyi J: Are ICD-10 codes appropriate for performance assessment in asthma and COPD in general practice? Results of a cross sectional observational study. BMC Health Serv Res 2005, 5:11.

20. Wensing M, Oxman A, Baker R, Godycki-Cwirko M, Flottorp S, Szecsenyi J, Grimshaw J, Eccles M: Tailored implementation for chronic diseases (TICD): A project protocol. Implementation Science 2011, 6:103.

21. Donner A, Klar N: Design and Analysis of Cluster Randomization Trials in Health Research. London: Arnold; 2000. 
22. Eccles M, Grimshaw J, Campbell M, Ramsay C: Research designs for studies evaluating the effectiveness of change and improvement strategies. Qual Saf Health Care 2003, 12:47-52.

23. Campbell MK, Thomson S, Ramsay CR, MacLennan GS, Grimshaw JM: Sample size calculator for cluster randomized trials. Comput Biol Med 2004, 34:113-125.

24. Murphy K, Casey D, Devane D, Cooney A, McCarthy B, Mee L, Nichulain M, Murphy AW, Newell J, O' Shea E: A cluster randomized controlled trial evaluating the effectiveness of a structured pulmonary rehabilitation education programme for improving the health status of people with chronic obstructive pulmonary disease (COPD): the PRINCE study protocol. Bmc Pulm Med 2011, 11:4.

25. Smeeth L, Ng ESW: Intraclass correlation coefficients for cluster randomized trials in primary care: data from the MRC trial of the assessment and management of older people in the community. Control Clin Trials 2002, 23:409-421.

26. Marcinowicz L, Chlabicz S, Grebowski R: Open-ended questions in surveys of patients' satisfaction with family doctors. J Health Serv Res Policy 2007, 12:86-89.

27. Muras M, Krajewski J, Nocun M, Godycki-Cwirko M: A survey of patient behaviours and beliefs regarding antibiotic self-medication for respiratory tract infections in Poland. Arch Med Sci 2013, 9(5):854-857. doi:10.5114/aoms.2012.29217.

28. Prakash A, Risser RC, Mallinckrodt CH: The impact of analytic method on interpretation of outcomes in longitudinal clinical trials. Int J Clin Pract 2008, 62:1147-1158.

29. Flottorp SA, Oxman AD, Krause J, Musila NR, Wensing M, Godycki-Cwirko M, Baker R, Eccles MP: A checklist for identifying determinants of practice: a systematic review and synthesis of frameworks and taxonomies of factors that prevent or enable improvements in healthcare professional practice. Implementation Science 2013, 8:35.

30. Wilson DH, Wakefield MA, Steven ID, Rohrsheim RA, Esterman AJ, Graham NM: "Sick of Smoking": evaluation of a targeted minimal smoking cessation intervention in general practice. Med J Aust 1990, 152:518-521. http://www.goldcopd.org/uploads/users/files/GOLD_Report_2013_Feb20.pdf.

31. Fletcher $\mathrm{CM}$, Elmes $\mathrm{PC}$, Fairbairn MB, Wood CH: The significance of respiratory symptoms and the diagnosis of chronic bronchitis in a working population. BMJ 1959, 2:257-266. http://www.nice.org.uk/ nicemedia/live/13029/49397/49397.pdf.

32. Qaseem A, Wilt TJ, Weinberger SE, Hanania NA, Criner G, van der Molen T, Marciniuk DD, Denberg T, Schünemann H, Wedzicha W, MacDonald R, Shekelle P, for the American College of Physicians; American College of Chest Physicians, the American Thoracic Society and European Respiratory Society: Diagnosis and management of stable chronic obstructive pulmonary disease: a clinical practice guideline update from the American College of Physicians, American College of Chest Physicians, American Thoracic Society and European Respiratory Society. Annals of Internal Medicine 2011, 155:179-191.

33. Gorecka D, Jassem E, Pierzchala W, Sliwinski P: Zalecenia Polskiego Towarzystwa Chorób Płuc dotyczące rozpoznawania i leczenia przewlekłej obturacyjnej choroby płuc (POChP [Recommendations of the polish society of lung diseases for diagnosis and treatment of chronic obstructive pulmonary disease (COPD)]. Pneumonol Alergol Pol 2012, 80:220-254

34. Jager CJ, Freund T, Steinhäuser J, Aakhus E, Flottorp S, Godycki-Cwirko M, van Lieshout J, Krause J, Szecsenyi J, Wensing M: Tailored Intervention for Chronic Diseases (TICD): a protocol for process evaluation in cluster randomized controlled trials in five European countries. Trials 2014, 15:87. doi:10.1186/1745-6215-15-87.

35. Schermer TR, Akkermans RP, Crockett AJ, van Montfort M Grootens-Stekelenburg J, Stout JW, Pieters W: Effect of e-learning and repeated performance feedback on spirometry test quality in family practice: a cluster trial. Ann Fam Med 2011, 9:330-336.
36. Kennedy A, Bower P, Reeves D, Blakeman T, Bowen R, Chew-Graham C, Eden M, Fullwood C, Gaffney H, Gardner C, Lee V, Morris R, Protheroe J, Richardson G, Sanders C, Swallow A, Thompson D, Rogers A, Salford National Institute for Health Research Gastrointestinal programme Grant Research Group: Implementation of self management support for long term conditions in routine primary care settings: cluster randomized controlled trial. BMJ 2013, 346:f2882.

doi:10.1186/1745-6215-15-109

Cite this article as: Godycki-Cwirko et al.: Evaluation of a tailored implementation strategy to improve the management of patients with chronic obstructive pulmonary disease in primary care: a study protocol of a cluster randomized trial. Trials 2014 15:109.

\section{Submit your next manuscript to BioMed Central and take full advantage of:}

- Convenient online submission

- Thorough peer review

- No space constraints or color figure charges

- Immediate publication on acceptance

- Inclusion in PubMed, CAS, Scopus and Google Scholar

- Research which is freely available for redistribution

Submit your manuscript at www.biomedcentral.com/submit
C) Biomed Central 